\title{
El agua corporal medida por impedancia eléctrica y su estimación según fórmulas convencionales $y$ en función del peso ideal y sexo, en adultos con sobrepeso u obesidad
}

Body water measured by electric bio impedance and its estimation by conventional formula based on ideal body weight and gender in overweight or obese adults

José Aarón Zapata Negreiros a , Joseph Arturo Gallardo Castro a, Juan Oscar Lluncor Vásquez ${ }^{\text {1,b; }}$, Javier Antonio Cieza Zevallos 1,c; 2,d

\section{RESUMEN}

Objetivos: Determinar el grado de precisión de las fórmulas antropométricas de Watson, Hume-Weyers, y la calculada en función del peso ideal y el sexo para estimar el agua corporal con la medida por impedancia eléctrica, en jóvenes con sobrepeso u obesidad. Material y métodos: Se enroló a 13 voluntarios sanos con edad entre 21 a 29 años, asintomáticos y con índice masa corporal (IMC) mayor de 25. Se evaluó la precisión en estimar el agua corporal en relación con la impedancia eléctrica de cada individuo con la t de Student apareada, y para estimar el error sistemático se utilizó análisis de regresión lineal. Resultados: El agua corporal promedio según la impedancia eléctrica fue 39,7 $\pm 6,49$ litros y según las fórmulas de Watson 42,66 $\pm 7,40$; Hume-Weyers 40,51 $\pm 6,31$, peso y sexo $45,90 \pm 8,80$ y peso ideal y sexo $39,90 \pm 6,10$ litros, respectivamente. La fórmula de Hume-Weyers y la estimación en función del peso ideal estimaron sin error significativo el agua medida por impedancia. Sin embargo, todas tuvieron buena correlación lineal con la impedancia. Conclusiones: La fórmula de Hume-Weyers y el cálculo del agua corporal según el peso ideal mostraron mayor precisión en la estimación del agua corporal en jóvenes con sobrepeso y obesidad en relación con la medida por impedancia.

PALABRAS CLAVE: Agua corporal, antropometría, impedancia eléctrica. (Fuente: DeCS BIREME).

\section{SUMMARY}

Objectives: To determine the precision in estimating body water using the anthropometric formula of Watson's, Hume-Weyers', and ideal body water and gender, compared to that measured by electric bio impedance in overweight or obese young adults. Methods: 13 healthy volunteers with body mass index above 25 between 21 and 29 years were recruited. Paired students' t-test was used to evaluate the precision in the estimation of body water, and logistic regression analysis was used to evaluate systematic error. Results: Total body water measured by bio electric impedance was $39.7 \pm 6.49$ liters; $42.66 \pm 7.40$ liters by Watson's formula; $40.51 \pm 6.31$ liters by HumeWeyers' formula; $45.90 \pm 8.80$ liters by weight and gender, and $9.90 \pm 6.10$ liters by ideal body weight and gender. The Hume-Weyers' formula and ideal body weight estimated with no significant error the body weight measured

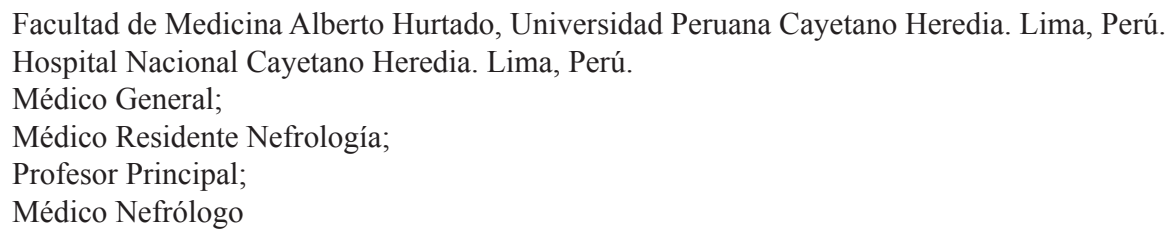


by electric bio impedance. However, all had good correlation with impedance. Conclusions: The Hume-Weyers' formula and ideal body weight showed better precision in estimating body water in young overweight or obese adults compared to bio impedance.

KEYWORDS: Body water, anthropometry, electrical impedance. (Source: MeSH NLM).

\section{INTRODUCCIÓN}

El agua es el elemento más abundante en el cuerpo y se mantiene constante en personas sanas. Pero se altera cuando existen condiciones que afectan el medio interno como las enfermedades crónicas y ciertos estilos de vida, además de las alteraciones hidroeléctricas, las hemorragias y la insuficiencia renal (1).

La alteración del agua corporal total afecta en gran medida a la salud como se observa en la práctica clínica, por ello es importante tener una aproximación certera del agua corporal total como patrón referencial del estado de salud o enfermedad. Es importante conocer el agua corporal en la terapia con soluciones parenterales, correcciones hidroelectrolíticas y del estado ácido-base, evaluaciones farmacocinéticas y valoración del estado de salud, entre otros.

El método más exacto para la determinación del agua corporal es mediante el uso de dióxido de deuterio diluido $(2,3)$, pero requiere tiempo y es de alto costo (3). Otro método validado es el de la impedancia eléctrica; esta técnica se fundamenta en la resistencia de las células al paso de una corriente eléctrica. La masa magra o libre de grasa, que contiene la mayoría de fluidos y electrolitos corporales, es un buen conductor eléctrico, es decir presenta baja impedancia, mientras que la grasa que actúa como un aislante, presenta alta impedancia $(2,4)$.

Estos estudios sin embargo, por ser relativamente costosos o de difícil uso, son poco accesibles a las poblaciones en vías de desarrollo como la nuestra. Por esta razón, se han desarrollado fórmulas para estimar el agua corporal usando las medidas antropométricas de la persona. Dentro de ellas tenemos las fórmulas de Watson (5) y de Hume-Weyers (6), que fueron desarrolladas utilizando métodos de regresión lineal en población sin alteración hídrica clínica evidenciable. Sin embargo, Basile (7) mostró que las ecuaciones antropométricas para la estimación del agua corporal total sólo debían utilizar en poblaciones específicas para evaluar diferencias individuales, no para comparaciones entre poblaciones.
Estas fórmulas no han sido validadas en nuestras poblaciones, cuyas características de sobrepeso $\mathrm{u}$ obesidad pueden ser diferentes a las de la población utilizada para el desarrollo de las fórmulas, y deberíamos saber cuál es la confianza de usarlas. En nuestro medio se usa solamente el cálculo del agua corporal de acuerdo a una proporción fija del peso: $60 \%$ para varones y $50 \%$ para mujeres y gerontes. Si bien esto puede ser muy operativo para la práctica clínica diaria, no sabemos si subestima o sobrestima los valores reales del agua corporal en personas con un IMC mayor de 25, considerando éste como el máximo valor normal.

Entonces surge la pregunta, si en personas aparentemente sanos pero con sobrepeso u obesidad, las fórmulas de Watson y Hume-Weyers y el cálculo utilizando proporciones fijas del peso en función del sexo y edad, son o no exactas en comparación con la impedancia eléctrica en una población como la nuestra. Se incorpora la hipótesis si el estimar el agua corporal ajustándola al peso máximo ideal de la persona (considerando un IMC en 25) permite inferir el agua corporal sin mayor error en relación a la impedancia en este tipo de personas. Esta consideración fue establecida asumiendo que en la persona joven y aparentemente sana, el contenido del agua corporal debería ser comparable al de otra persona con un IMC de 25 como valor máximo $(9,10)$.

El objetivo del estudio fue determinar el grado de precisión de las diferentes formas de estimar el agua corporal en una muestra de jóvenes sanos entre 20 y 30 años pero con sobrepeso u obesidad.

\section{MATERIAL Y MÉTODOS}

Estudio observacional, comparativo auto controlado realizado en voluntarios sanos con edad entre 21 y 29 años e IMC $>25$, estudiantes de Medicina de la Facultad de Medicina Alberto Hurtado. Se definió voluntario sano aquella persona asintomática, sin evidencia de trastornos hemodinámicos, ni trastornos hídricos quien debería haber pasado su examen médico anual programado sin habérseles detectado anormalidades. 
Se calculó el tamaño de muestra a partir de la fórmula que considera muestras apareadas con desviación estándar de acuerdo a estándares ya definidos (8). Para nuestro caso, se usaron los valores de desviación estándar igual a 0,05 (considerando que los datos corresponden a datos apareados en una distribución mesocúrtica de la población blanco del estudio), $\alpha$ de 0,05 (confianza del 95\%), $\beta 0,8$ (potencia igual a $80 \%$ ) y una $\Delta$ (diferencia) de 0,05 elegido arbitrariamente, que significa el punto a partir del cual las diferencias observadas tienen relevancia clínica. El tamaña de muestra al aplicar la fórmula resultó en 10. La técnica de selección de voluntarios fue por muestreo por conveniencia.

Para la recolección de datos, se entrevistó a cada voluntario explicando el objetivo y procedimiento a realizar y se entregó una ficha informativa. Luego de la aceptación verbal de participar en el estudio, se procedió a la medición de peso y talla en condiciones estándar usando una balanza de pie previamente calibrada. Solamente fueron incluidos personas cuyo IMC era mayor de 25. En ellos se midió el perímetro de la cintura y de la cadera tomando como punto de reparo para el perímetro de la cintura la última costilla flotante y para la cadera, los glúteos.

Para la medición de la impedancia eléctrica se utilizó la máquina BODYSTAT ${ }^{\circledR} 1500$ MDD y se siguieron los procesos estandarizados internacionalmente $\mathrm{y}$ los recomendados por el fabricante, colocándose los electrodos a la altura de la cabeza del cúbito derecho, a la altura de la segunda articulación metacarpo falángica derecha, a la altura del tobillo derecho (entre ambos maléolos) y a la altura de la segunda articulación metatarso falángica derecha. Luego de introducir los datos de cada voluntario, se le instruyó para mantenerse tranquilo e inmóvil. Una vez efectuada la primera medición, se tomó nota del valor de agua corporal tanto en porcentaje como en volumen expresado en litros. Finalmente, se retiró los cables y electrodos del sujeto en estudio y se dio por concluido el procedimiento.

Los datos fueron almacenados de manera anónima en una hoja de cálculo electrónica y para el análisis de datos se utilizó el programa informático SPSS Statistics v. 19.

Simultáneamente otro investigador con desconocimiento de los resultados de la medición del agua corporal por el método de impedancia, calculó el agua corporal total según las fórmulas de Watson,
Hume-Weyers, la que asume el $60 \%$ del peso medido para varones y $50 \%$ para mujeres (P1) y la propuesta de inferir el agua corporal en función del peso ideal asumido de un IMC de 25, de la siguiente manera:

a) Fórmula de Watson en varones $=2,447-(0,09156$ $\mathrm{x}$ edad $)+(0,1074 \mathrm{x}$ talla $)+(0,3362 \mathrm{x}$ peso $)$

b) Fórmula de Watson en mujeres $=-2,097+(0,1069$ $\mathrm{x}$ talla $)+(0,2466 \mathrm{x}$ peso $)$

c) Fórmula de Hume en varones $=(0,194786 \mathrm{x}$ talla $)+(0,296785 \times$ peso $)-14,012934$

d) Fórmula de Hume en mujeres $=(0,34454 \times$ talla $)$ $+(0,183809 \times$ peso $)-35,270121$

e) Fórmula función del peso y sexo $=(\mathrm{K} \times \mathrm{Peso})$; donde $\mathrm{K}=0,6$ si es varón y 0,5 si es mujer

f) Fórmula función del peso ideal y sexo $=(\mathrm{K} x$ Peso ideal); donde $\mathrm{K}=0,6$ si es varón y 0,5 si es mujer. (El peso ideal se calculó considerando un IMC de 25 y la talla de cada persona según: Peso ideal $=$ $\left.\left[\operatorname{talla}^{2}(\mathrm{~m}) \times 25\right]\right)$.

Para el análisis de los datos se utilizó la t de Student apareada para comparar los promedios de la diferencia obtenida para cada sujeto entre el agua corporal estimada en cada fórmula y el valor obtenido por impedancia. Se aceptó una significancia estadística de $\mathrm{p}<0,05$ para rechazar la hipótesis nula. Finalmente, se realizó el análisis de regresión lineal considerando como variable independiente el agua corporal medida por la impedancia eléctrica y como variable dependiente el agua corporal estimada según cada formulación estimada.

El estudio fue revisado y aprobado por el Comité Institucional de Ética de la Universidad Peruana Cayetano Heredia.

Tabla 1. Valor del agua corporal medido por Impedancia eléctrica y estimado según las fórmulas propuestas en jóvenes con IMC $>25$ (10 varones, 3 mujeres).

\begin{tabular}{lc}
\hline Método & $\begin{array}{c}\text { AGUA CORPORAL } \\
\text { (Litros) }\end{array}$ \\
\hline Impedancia & $39,70 \pm 6,48$ \\
Fórmula de Watson & $42,66 \pm 7,40$ \\
$\begin{array}{l}\text { Según Porcentaje del peso y } \\
\text { sexo }\end{array}$ & $45,90 \pm 8,80$ \\
$\begin{array}{l}\text { Según Fórmula Hume } \\
\text { Según Porcentaje del peso } \\
\text { ideal y sexo }\end{array}$ & $40,51 \pm 6,31$ \\
\hline
\end{tabular}




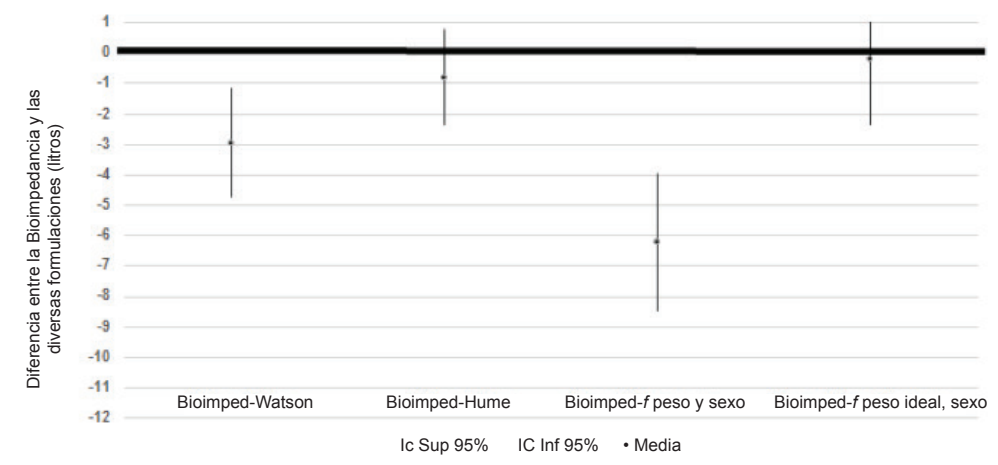

Gráfico 1. Diferencia observada del valor agua corporal medida por impedancia eléctrica y su estimación según las diversas fórmulas propuestas para cada persona estudiada.

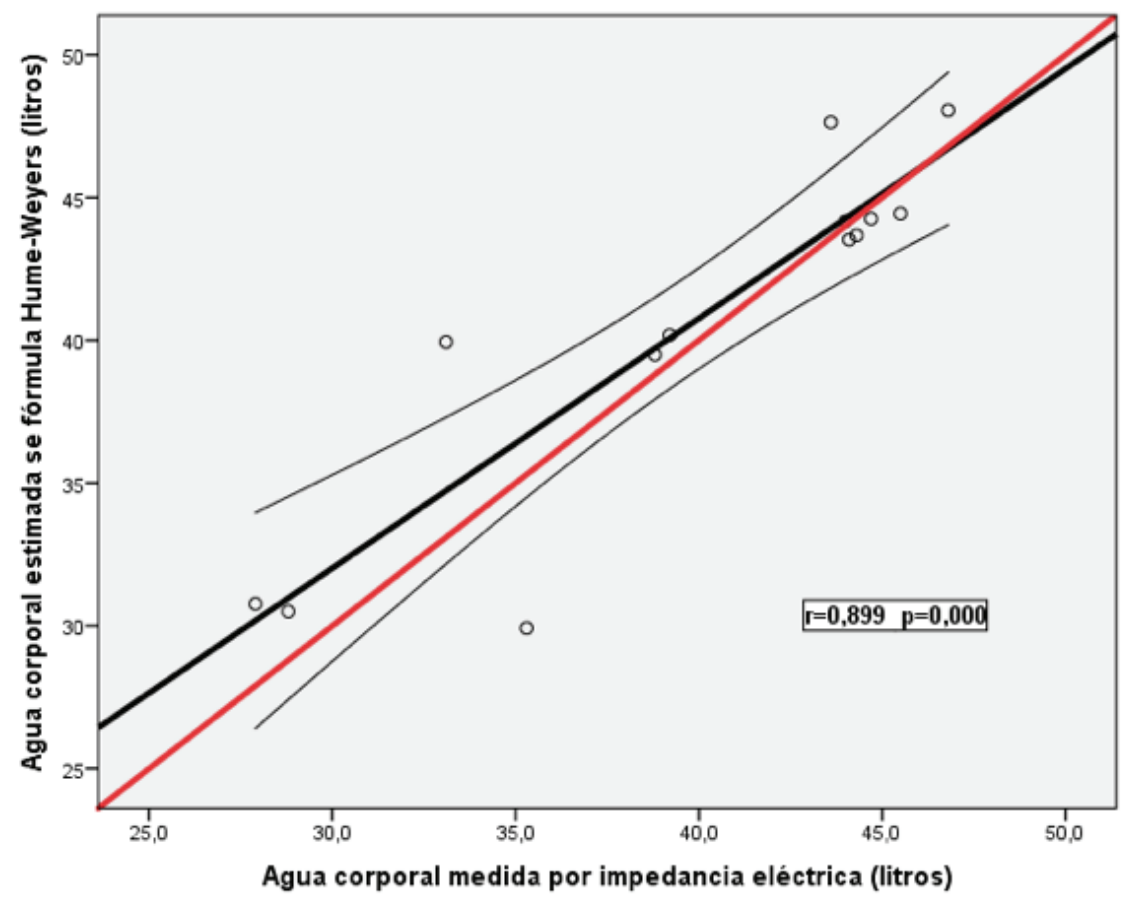

Nota: La linea roja representa la línea de identidad

Gráfico 2. Correlación lineal del agua corporal medida por Impedancia eléctrica y su estimación según la fórmula de Hume-Weyers en sujetos jóvenes con sobrepeso u obesidad.

\section{RESULTADOS}

Los voluntarios estudiados fueron 13, (10 varones y 3 mujeres), el promedio de edad fue $23,9 \pm 1,63$ años, talla $167,20 \pm 8,70 \mathrm{~cm}$, peso $70,78 \pm 12,11 \mathrm{~kg}$, superficie corporal $1,73 \pm 0,16 \mathrm{~m}^{2}$ e IMC $28,73 \pm 3,40$ $\mathrm{kg} / \mathrm{m}^{2}$. En la tabla 1 se presentan los resultados de las mediciones del agua corporal según la Impedancia eléctrica y según las diferentes fórmulas propuestas para su estimación de los sujetos estudiados y según el sexo. No hubo diferencia estadística entre los promedios según las diversas fórmulas y la medida por impedancia.

El gráfico 1 muestra la diferencia observada individualmente del agua corporal calculada por impedancia y las diferentes fórmulas. Las fórmula de Watson y la que estima el agua en función del peso actual y sexo sobrestimaron el agua corporal medida por impedancia. La fórmula de Hume-Weyers y la 


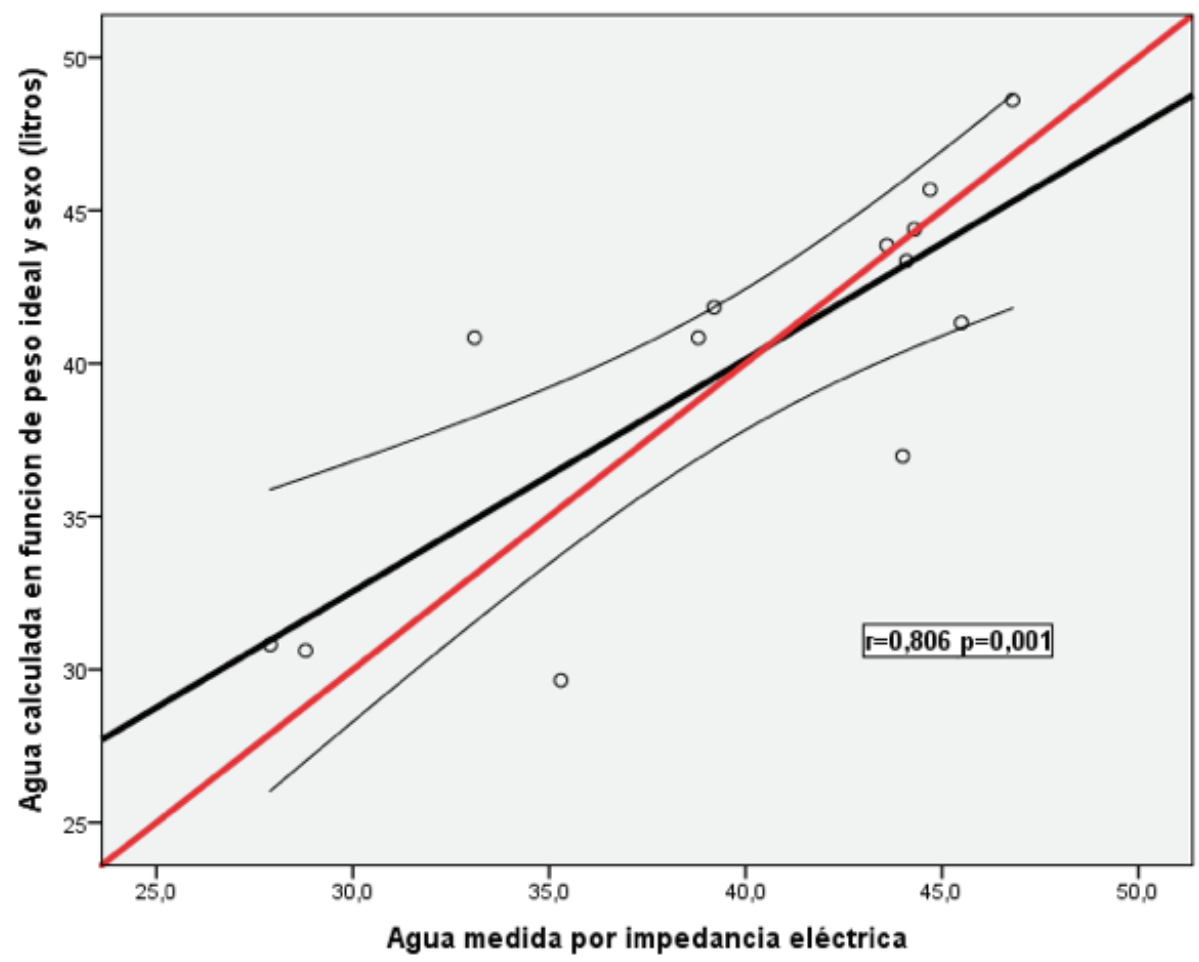

Nota: La linea roja representa la línea de identidad

Gráfico 3. Correlación lineal del agua corporal medida por Impedancia eléctrica y su estimación según el peso ideal y sexo en sujetos jóvenes con sobrepeso u obesidad.

estimada mediante el cálculo según el peso ideal y sexo de cada persona, no mostraron diferencia significativa en relación a la medida por impedancia.

La correlación entre la medición del agua corporal por impedancia eléctrica y la estimada por las diversas fórmulas fue: $\mathrm{r}=0,896$ para la fórmula de Watson y $\mathrm{r}=0,899$ para la de Hume-Weyers; para la estimación del agua corporal en función del porcentaje del peso corporal de la persona y sexo fue $r=0,894(p=0,000)$ y $r=0,806(p=0,001)$ para el agua corporal estimada en función del porcentaje del peso corporal ideal de la persona y sexo. El gráfico 2 muestra la correlación del agua corporal medida por impedancia y la fórmula de Hume-Weyers y el gráfico 3 presenta la correlación del agua corporal medida por impedancia y la calculada en función del peso ideal y sexo de la persona.

\section{DISCUSIÓN}

El objetivo del estudio fue evaluar la precisión de las fórmulas antropométricas en la estimación del agua corporal total en nuestra población joven, aparentemente sana pero con sobrepeso. Los resultados muestran que si bien todas las propuestas tienen valores promedio similares, al contrastar en cada sujeto el valor obtenido por la impedancia y con cada fórmula, la propuesta de Watson y la estimada en función del peso y sexo, mostraron una sobrestimación significativa del agua corporal, situación no observada con la fórmula de Hume-Weyers y la estimada en función del peso ideal y sexo como se aprecia en el gráfico 1.

Johansson (11), mostró que las fórmulas antropométricas tienden a sobreestimar el agua corporal total en pacientes obesos en diálisis peritoneal y en personas con sobrehidratación, y tiende a subestimarla en delgados.

La fórmula de Hume-Weyers propuesta en el año 1971 recolectó voluntarios de un hospital inglés, pacientes sin evidencia de retención de fluidos ni malnutrición; para la formulación de Watson en 1980 se recolectó la información a partir de las publicaciones existentes hasta esa fecha en la literatura mundial (excluyendo la asiática) sobre agua corporal total, recabando la edad, sexo, peso y talla de los sujetos incluidos en estos estudios (5).

La determinación del agua corporal en función del porcentaje del peso corporal y el sexo sobreestimó 
el agua corporal y no debería usarse en este tipo de personas a menos que se considere el peso ideal que ha mostrado tan buena precisión, como la fórmula de Hume-Weyers, aunque con un error sistemático discretamente mayor.

Nuestro estudio permite concluir que la fórmula de Hume-Weyers para estimar el agua corporal es la que ha mostrado mayores ventajas aun en situaciones de sobrepeso y obesidad. Aunque si se estima el agua corporal en función del peso ideal y sexo, también puede considerarse totalmente válido, no así si se estima con el peso real de la persona.

No se debe inferir que esto es válido para personas con edad avanzada, para quienes deben hacerse las observaciones pertinentes.

\section{Declaración de financiamiento y de conflictos de intereses:}

El estudio fue financiado por los autores, declaran no tener conflictos de interés.

\section{Contribución de autoría:}

JAZN，JAGC，JOLV y JACZ, participaron en el diseño del estudio, recolección de los datos, interpretación de los resultados y aprobación de la versión a ser publicada.

\section{Correspondencia:}

Javier Cieza Zevallos

javier.cieza@upch.pe

\section{REFERENCIAS BIBLIOGRÁFICAS}

1. Lee S, Song J, Kim G, Lee K, Kim M. Assessment of total body water from anthropometry-based equations using bioelectrical impedance as reference in Korean adult control and haemodialysis subjects. Nephrol Dial Transplant. 2001; 16(1):91-7.
2. Woodrow G, Oldroyd B, Wright A, Coward W, Truscott J. Comparison of anthropometric equations for estimation of total body. Nephrol Dial Transplant. 2003; 18(2):384-389.

3. Sanchez Jager A, Adela Baron M. Uso de la impedancia eléctrica para la estimación de la composición corporal en niños y adolescentes. An Venez Nutr. 2009; 22(2):105-110.

4. Piers L, Soares M, Frandsen S, O'Dea K. Indirect estimates of body composition are useful for groups but unreliable in individuals. Int J Obes Relat Metab Disord. 2000; 24(9):1145-1152.

5. Watson P, Watson I, Batt R. Total body water volumes for adult males and females estimated from simple anthropometric measurements. Am J Clin Nutr. 1980; 33(1):27-39.

6. Hume R, Weyers E. Relationship between total body water and surface area in normal and obese subjects. J Clin Pathol. 1971; 24(3):234-8.

7. Basile C, Vernaglione L, Bellizzi V, Lomonte C, Rubino A, D'Ambrosio N, et al. Total body water in health and disease: Have anthropometric equations any meaning? Nephrol Dial Transplant. 2008; 23(6):1997-2002.

8. Salkind NJ. Muestreo y generalizabilidad. En: Salkind NJ. Métodos de investigación. 3rd ed. México: Prentice Hall; 1999.p. 400.

9. Altman P. Blood and other body fluids. Washington: Federation of American Societies for Experimental Biology; 1961.

10. Brouns F. Necesidades nutricionales de los atletas. Barcelona: Editorial Paidotribo; 2001.p. 67.

11. Johansson AC, Samuelsson O, Attman PO, Bosaeus I, Haraldsson B. Limitations in anthropometric calculations of total body water in patients on peritoneal dialysis. J Am Soc Nephrol. 2001; 12(3): 568-573.
Recibido: 01/03/2016

Aceptado: 30/06/2016 